\title{
Mapping Stacking and Stacking Defects in the 2D Ferromagnet CrI3
}

Ariana Ray, Yu-Tsun Shao, Yang Xu, Nikhil Sivadas, Tingxin Li, Zefang Wang, Kin Fai Mak, Jie Shan, Craig Fennie and David Muller

Cornell University, Ithaca, New York, United States

Different lateral shifts of the stacked monolayers in $\mathrm{CrI}_{3}$ lead to different magnetic ground states. A rhombohedral-symmetry interlayer stacking, generated by $(1 / 3,2 / 3)$ fractional unit cell shifts, is predicted to result in ferromagnetic layer ordering, while a monoclinic-symmetry interlayer stacking, whose variants are created by $2 / 3$ unit cell shifts along $\mathrm{Cr}$-I bond directions, leads to antiferromagnetic ordering $[1,2]$. Though the structure of bilayer $\mathrm{CrI}_{3}$ has been inferred from second harmonic generation (SHG) [3], the structure of few-layer $\mathrm{CrI}_{3}$ remains relatively unstudied, due to the complications that the increased structural diversity presents to macroscopically averaged techniques, and the air and radiation-sensitivity of the material in its $2 \mathrm{D}$ form. To prevent rapid degradation, we typically encapsulate few-layer $\mathrm{CrI}_{3}$ in hBN of comparable or greater thickness, which decreases the sample-to-background ratio and prevents atomic-resolution imaging, but still allows diffraction studies with a high dynamic range detector [4].

Here we explore the stacking arrangements found in $2 \mathrm{D} \mathrm{CrI} 3$ as the thickness is systematically increased from monolayer to the few-layer regime, using atomic-resolution imaging and 4D-STEM electron diffraction. We find that $\mathrm{CrI}_{3}$ flakes from bilayer to $\sim 15 \mathrm{~nm}$ thickness remain in a monoclinic stacking when cooled to $93 \mathrm{~K}$. However, the monoclinic stacking allows for multiple variants - arising from inlayer and interlayer $120^{\circ}$ stacking rotations - and we observe a rich assortment of stacking orders and domains, as well as moirés, from combinations of these variants.

Figure 1 gives atomic-resolution evidence of multiple monoclinic rotation variants in room-temperature, few-layer $\mathrm{CrI}_{3}$. The monoclinic space group can be uniquely identified in top-down atomic-resolution images by a striped pattern from layers of Chromium atoms shifted in-plane along one of the three Cr-I bond directions, forming lines in projection. The families of stripes, separated by rotations of $120^{\circ}$, correspond to different monoclinic variants. In Figure 1, experimental high angle annular dark field (HAADF) images and their diffractograms are matched to multislice simulations from monoclinic structures containing one rotational variant (Fig. 1a, c) and a vertical mixture of two variants (Fig. 1b, d). The number of stripe families in the HAADF image, or equivalently the number of first-order spots in the diffractogram/diffraction pattern, reveals the number of monoclinic variants present.

We observe that stacking domains of monoclinic variants can change as a function of temperature. Figure 2 shows dark field images from 4D-STEM scans of few-layer $\mathrm{CrI}_{3}$ at different temperatures: initial $293 \mathrm{~K}$ (Fig. 2a), cooled to $93 \mathrm{~K}$ (Fig. 2b), and reheated to $293 \mathrm{~K}$ (Fig. 2c). In each image, the bright domains contain the so-defined $0^{\circ}$ monoclinic variant, while the dark regions contain the $\pm 120^{\circ}$ variants. The pair of outlined domains diminishingly alternate in contrast as the sample is cooled and then heated, indicating that their stacking transitions from the $0^{\circ}$ to the $\pm 120^{\circ}$ monoclinic variants before settling in a mixture of the three. The left side of Fig. 2a also shows a region with moiré, caused by an additional twist angle of $\sim 1.1^{\circ}$ between two monoclinic rotation variants.

Surprisingly, the structural phase transition to rhombohedral interlayer stacking - believed to occur in the bulk below $220 \mathrm{~K} \mathrm{[2]-is} \mathrm{not} \mathrm{observed} \mathrm{in} \mathrm{any} \mathrm{of} \mathrm{the} \mathrm{2D} \mathrm{CrI3} \mathrm{samples} \mathrm{up} \mathrm{to} 50 \mathrm{~nm}$ in thickness. Instead, we note that the observed superposition of monoclinic variants in few-layer $\mathrm{CrI}_{3}$ flakes may present as an 
overall three-fold symmetry to macroscopically averaged techniques such as SHG, but can be resolved by atomic-resolution and nanodiffraction imaging. In contrast, the few-layer $\mathrm{CrBr}_{3}$ system, isoelectronic to $\mathrm{CrI}_{3}$ but with a higher bulk monoclinic-to-rhombohedral stacking transition temperature of $\sim 420 \mathrm{~K}[5,6]$, shows the expected room-temperature rhombohedral stacking when examined by 4D-STEM, even though in several areas stacking domains due to mixtures of monoclinic variants are also found. [7]
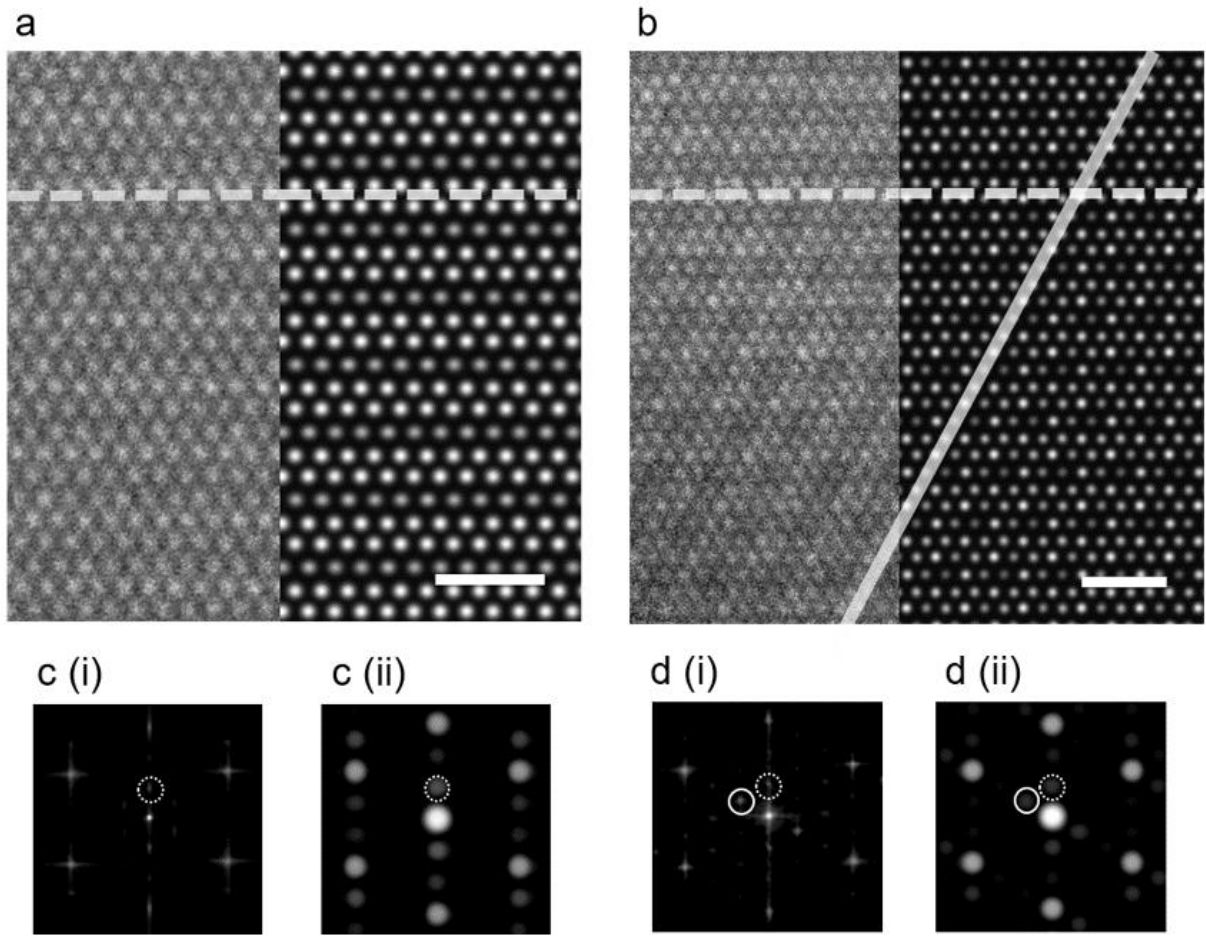

Figure 1. (a), (b) Left sides: Experimental top-down HAADF images from few-layer CrI3 at room temperature, showing respectively a single domain and a region containing overlapping monoclinic variants. The single-variant image (a) has only one stripe family, while the two-variant image (b) has two sets of stripes (dashed/solid lines). (a), (b) Right sides: Matching HAADF simulations from CrI3 with (a) only one monoclinic variant and (b) a vertical mixture of two monoclinic variants $120^{\circ}$ apart. (c), (d) i: Diffractograms from the experimental HAADF images. (c), (d) ii: Diffraction simulations of the same one-variant and two-variant monoclinic structures from (a) and (b), respectively. The circled first-order diffraction spots from the monoclinic stripes correspond to the circled peaks in the diffractograms, and the dashed/solid circle outlines match the corresponding real-space stripe families in (a), (b). Scale bars: $1 \mathrm{~nm}$. 

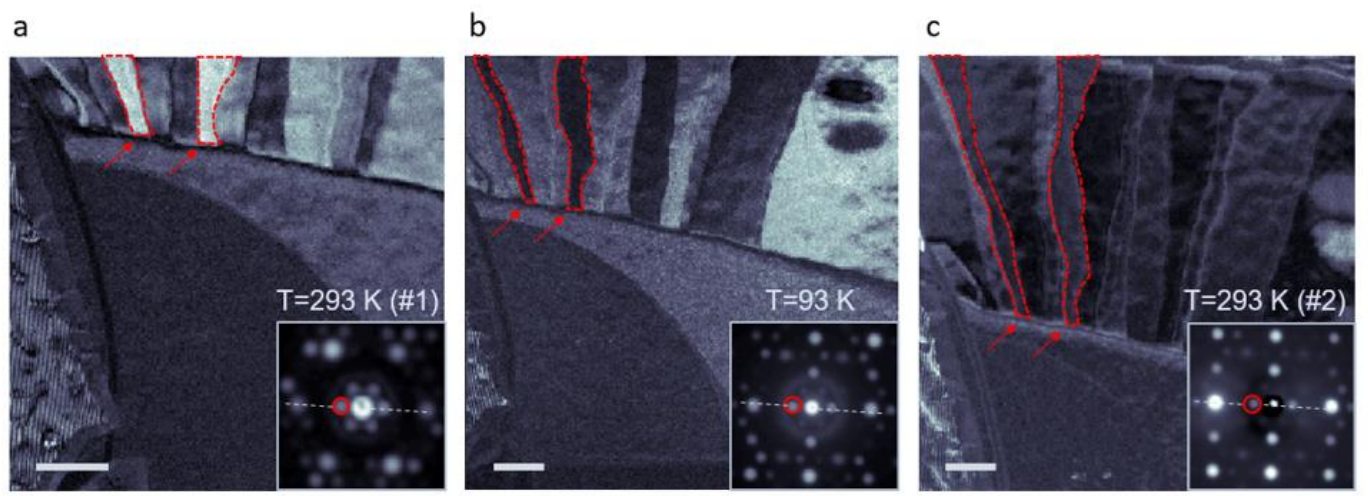

Figure 2. Dark field images of domains in few-layer CrI3 from the same diffraction peak (circled in insets) showing irreversible domain restructuring as temperature is cycled from: (a) initial $293 \mathrm{~K}$, to (b) $93 \mathrm{~K}$, and then again to (c) $293 \mathrm{~K}$. The white regions contain the $0^{\circ}$ monoclinic variant, which tracks with the circled diffraction spot, while the dark regions contain the $\pm 120^{\circ}$ variants. The same pair of domains is outlined in red on (a)-(c). As the temperature cycles, the outlined domains alternate contrast, indicating that their stacking transitions between different rotation variants. Note that in (b) and (c) the sample has been tilted away from the top-down zone axis about the dashed line drawn on the diffraction patterns in (a)-(c). Scale bars: $500 \mathrm{~nm}$.

\section{References}

[1] N. Sivadas, et al., "Stacking-Dependent Magnetism in Bilayer CrI3", Nano Lett. 2018, 18, 12, 76587664

[2] M. A. McGuire, et al., "Coupling of Crystal Structure and Magnetism in the Layered, Ferromagnetic Insulator $\mathrm{CrI}_{3}$ ", Chem. Mater. 2015, 27, 2, 612-620

[3] Z. Sun, et al., "Giant nonreciprocal second-harmonic generation from antiferromagnetic bilayer CrI3", Nature, 2019, 572, 497-501

[4] M. W. Tate, et al., "High Dynamic Range Pixel Array Detector for Scanning Transmission Electron Microscopy", Microsc. Microanal., 2016, 22, 1, 237-249

[5] M. A. McGuire, "Crystal and Magnetic Structures in Layered, Transition Metal Dihalides and Trihalides", Crystals, 2017, 7, 5, 121

[6] B. Morosin, et al., "X-Ray Diffraction and Nuclear Quadrupole Resonance Studies of Chromium Trichloride", 1964, 40, 7, 1958-1967

[7] Research supported by PARADIM DMR-1539918, AFOSR MURI FA9550-18-1-0480, CCMR DMR1719875 